\title{
Experimental Investigation on Coupled Cross-flow and In-line VIV Responses of Two Staggered Circular Cylinders
}

\author{
Cheng Zhang ${ }^{\text {a, b }}$, Zhuang Kang a , Yeping Xiong ${ }^{\mathrm{b}}$, Shangmao $\mathrm{Ai}^{\mathrm{a}}{ }^{*}$, Gang $\mathrm{Ma}^{\mathrm{a}}$ \\ (a College of Shipbuilding Engineering, Harbin Engineering University, Harbin 150001, China; b Faculty of Engineering \\ and Physical Sciences, University of Southampton, Southampton, SO17 1BJ, United Kingdom)
}

\begin{abstract}
In order to better understand the vortex-induced vibration (VIV) mechanism of multiple cylinders, this paper takes a relatively simple case of two staggered circular cylinders as the embarkation point, and investigates their VIV characteristics by model test. The experimental Reynolds number ranges from 22000 to 88000 . The inline gap $L$ is set as 3.0D, 3.6D, 4.2D and 5.5D in turn, and the cross-flow gap $T$ is set as $0.7 D, 1.1 D, 1.5 D, 1.9 D$, $2.3 D$ and $2.7 D$ respectively. By measuring the vibrating response in model test, the response differences between the two staggered cylinders and the isolated cylinder and the effects of the gaps are discussed. The results indicate that the variation trend of response of the upstream cylinder with reduced velocity is basically similar with that of the isolated cylinder. However, the downstream cylinder shows some great differences. When the in-line gap ratio $L / D$ is 3.6 , the cross-flow amplitude curve of downstream cylinder changes from "single peak" to "double peaks" with the increase of cross-flow gap ratio $T / D$, and in-line amplitude curve even shows four different kinds of forms. When $L / D$ is increasing, maximum amplitudes of the downstream cylinder in two directions also show an increasing trend, and the wake galloping phenomenon even appears in some conditions. Generally, the case of staggered cylinders is a generalized combination of two circular cylinders in tandem and side-by-side arrangements, and this paper has extended the research scope of the double-cylinder VIV to arbitrary flow direction.
\end{abstract}

Key words: staggered circular cylinders; vortex-induced vibration; model test; in-line and cross-flow gaps; amplitude response

\section{Introduction}

In the field of ocean engineering, the cylindrical structure is one of the most widely used structural forms, and many structures can be regarded as the combinations of double or multiple circular cylinders, such as a section of oil and gas pipe bundle, a group of adjacent buoyancy cans, several columns of a cell Spar and so on. The continuous generation and shedding of the vortex will produce the fluctuating lift and drag, which will further lead to the cross-flow and in-line VIV of the cylindrical structures. ${ }^{1}$ The VIV of a structure is one of the key issues of the structure's design because VIV increases the dynamic load on the structure and may cause fatigue damage. ${ }^{2}$ Due to the interference of the flow between the cylinders, the characteristics of the 
multiple-cylinder VIV are significantly different with those of an isolated circular cylinder, which may result in more complex problems about the response prediction and potential fatigue damage of multiple-cylinder structures. ${ }^{3}$ In order to better understand the VIV mechanism of a group of cylinders, many researchers often take the VIV of a pair of circular cylinders as the starting point.

Numerical simulation is generally considered as a kind of useful approach on the investigations of VIV about a pair of circular cylinders. Prasanth and $\mathrm{Mittal}^{4}$ carried out the numerical simulation of the vortexinduced vibration of a pair of equal-sized circular cylinders in tandem and staggered arrangements in laminar flow regime. They found the upstream cylinder in both tandem and staggered arrangements were qualitatively similar to a single cylinder while the downstream cylinder showed the large amplitudes at almost all reduced velocities. According to the numerical research of two tandem cylinders, affected regime of double tandem cylinders was re-classified by Carmo et al. ${ }^{5}$ as three main flow patterns: (i) When $\mathrm{L} / \mathrm{D}<1.5$, no swirl appears between two cylinders and the shear layer does not rotate (ii) When $\mathrm{L} / \mathrm{D}=1.8-2.3$, alternating swirl appears in the gap and the shear layer rotates (iii) When L/D $>5$, complete wake vortex appears in the gap. Derakhshandeh et al. ${ }^{6}$ analyzed the effect of arrangement of two circular cylinders on the maximum efficiency of VIV power using the Scale-Adaptive Simulation model, in which the upstream cylinder was fixed while the downstream was mounted elastically with one degree of freedom, and observed that cylinders offset from one another showed a higher efficiency compared to cylinders with their centres aligned. Chen et al. ${ }^{7}$ studied the cross-flow VIV characteristics of two elastically supported side-by-side circular cylinders with a center-to-center spacing ratio $T / D$ ranging from 2 to 5 in numerical simulation. They argued that the proximity of the cylinders did not have a significant impact to the lock-in region and cylinder responses, except at a small spacing ratio of $T / D=2$. Moreover, many other scholars, such as $\mathrm{Zhao}^{8}$, Tu et al. ${ }^{9}$, Mysa et al. ${ }^{10}$, and so on, all performed the numerical studies on the VIV mechanism as well as characteristics of double circular cylinders with different arrangements and obtained some significant and useful achievements.

Although the above-mentioned scholars have systematically studied the VIV characteristics of double cylinders by using numerical approaches, which broadens people's understanding to the complex phenomenon of VIV of a pair of cylinders to a great extent. However, both the phenomenon observation and mechanism exploration of the VIV and the verification of numerical simulation cannot be separated from the fundamental support of model test. 
Hover and Triantafyllou ${ }^{11}$ considered the effects of the upstream cylinder wake on the VIV response of the downstream cylinder in tandem VIV model test. They observed that, if a corrected reduced velocity is used which takes into account the wake flow conditions, some features of the downstream cylinder VIV can be explained as in single isolated cylinder VIV tests. Allen and Henning ${ }^{12}$ investigated two vertically tensioned long and flexible cylinders in tandem arrangement with the centre-to-centre distance varying from 3 to 12.5 cylinder diameters experimentally, and found that the downstream cylinder consistently experiences bimodal response even when the upstream cylinder response is dominated by a single mode. Assi et al. ${ }^{13}$ conducted the experiments on 1-DOF flow-induced vibration of two tandem circular cylinders to investigate their interference phenomenon, and found the trailing cylinder oscillation presented what previous researchers had termed interference galloping behavior for a centre-to-centre gap spacing ranging from 2D to 5.6D. Korkischko and Meneghini ${ }^{14}$ performed the experimental research on tandem circular cylinders fitted with strakes at a circulating water channel facility, in which the cylinders were restricted to oscillate in the transverse direction to the channel flow. By comparing with an isolated cylinder, they found that the suppression efficiency of the strakes was reduced due to the wake interference effect. Huang and Sworn ${ }^{15}$ carried out the model test of a pair of elastically-supported tandem cylinders of dissimilar diameters to investigate the hydrodynamic interaction between them, where the spacing between the two cylinders ranges from 1 to 10 times the larger cylinder diameter, and found that both the lift and the cross-flow motion of the downstream cylinder were influenced by the vortex shedding behind the two cylinders and the spacing between the two cylinders. Huera-Huarte and Gharib ${ }^{16}$ took a side-by-side arrangement of two vertical, high aspect ratio and low mass ratio cylinders as research object and preformed the VIV model test in a freesurface water channel. The cross-flow amplitudes, frequencies and phase synchronization of the models were investigated while the in-line vibration was neglected. Huang and Herfjord ${ }^{17}$ discussed the effects of the tandem and staggered positions on the forces and motion responses of two interfering VIV circular cylinders in model test, where the Reynolds numbers ranged from $1.12 \times 10^{4}$ to $5.52 \times 10^{4}$, and the reduced velocities were from 1.78 to 8.77 . Their work indicated the VIV response of the downstream cylinder was dependent upon the reduced velocity of the upstream cylinder and its own reduced velocity, besides, the motion response of the downstream cylinder was strongly influenced by the upstream cylinder in the spacing range tested. The above scholars all explored the interference phenomenon of double-cylinder VIV by conducting model 
tests, which provided basic contrast data for relevant numerical simulations.

In the relevant published research work, many scholars have been concentrated on the single-degree-offreedom VIV of a pair of tandem or side by side circular cylinders. The experimental investigations on the double-cylinder VIV with two degrees of freedom are fewer, in which, the case of two staggered cylinders is much rarer. Although Huang and Herfjord ${ }^{17}$ investigated the trajectories and amplitude characteristics of the VIV of two staggered cylinders with two degrees of freedom in model test, the reduced velocity was limited as 1.78 to 8.77 . However, the reduced velocities corresponding to the maximum value of up branch and the important values of lower branch in the two-degrees-of-freedom VIV of the cylinders with lower mass ratios are usually larger than 8 or $10 .{ }^{18}$ As a result, the characteristics of the VIV with two degrees of freedom about the staggered cylinders still need further explorations. Moreover, the staggered arrangement is a combination of the tandem and side-by-side arrangements, whose VIV characteristics may be closely associated with the cross-flow and in-line gaps, which also need more researching attentions.

Thus, in order to further develop the experimental research and explore the response characteristics of the two-degrees-of-freedom VIV of double staggered cylinders with higher reduced velocities corresponding to higher Reynolds numbers and provide the reference data for relevant numerical simulations, a set of experimental devices for the two-degrees-of-freedom VIV of two staggered circular cylinders are designed in this paper, following which a series of model tests for the two cylinders are conducted under different conditions of flow velocity and cross-flow and in-line gaps. On this basis, the differences of VIV characteristics between the isolated and staggered cylinders are discussed. Besides, the effects of cross-flow and in-line gap ratios on VIV amplitude responses are also explored systematically.

\section{Experimental set-up}

Tests were conducted at the towing tank of Harbin Engineering University (HEU). The towing tank facility at HEU have a $7 \times 3.5 \times 108 \mathrm{~m}$ test-section. The velocity range of the towing carriage is $0 \sim 6.5 \mathrm{~m} / \mathrm{s}$, and the stable precision is smaller than $0.1 \%$, which are controlled by computer program.

In this experiment, a set of testing auxiliary device was designed to ensure that the circular cylinders could vibrate with two degrees of freedom, whose design details are shown in Fig. 1. 


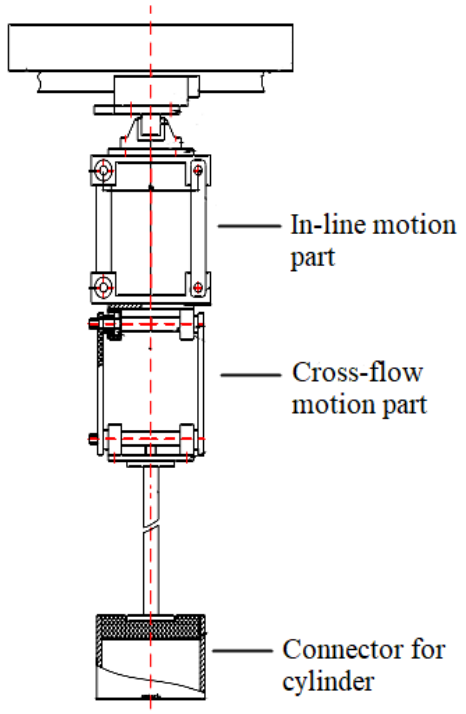

(a)

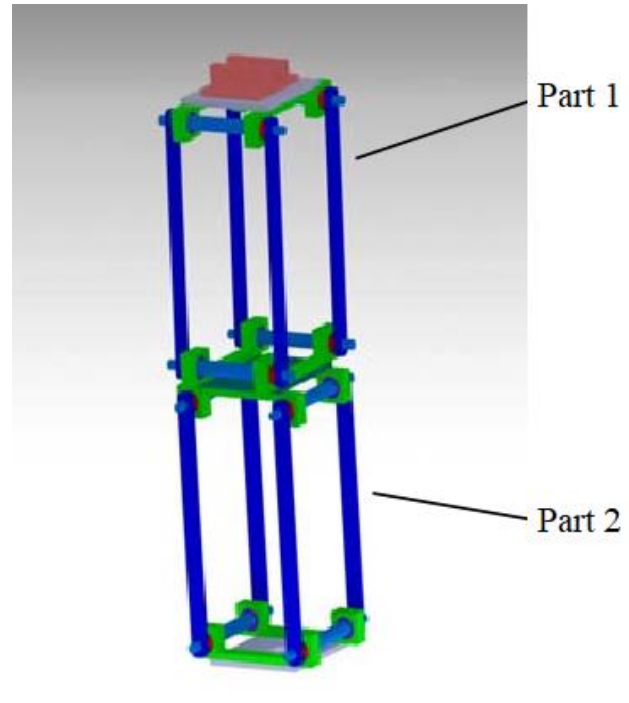

(b)

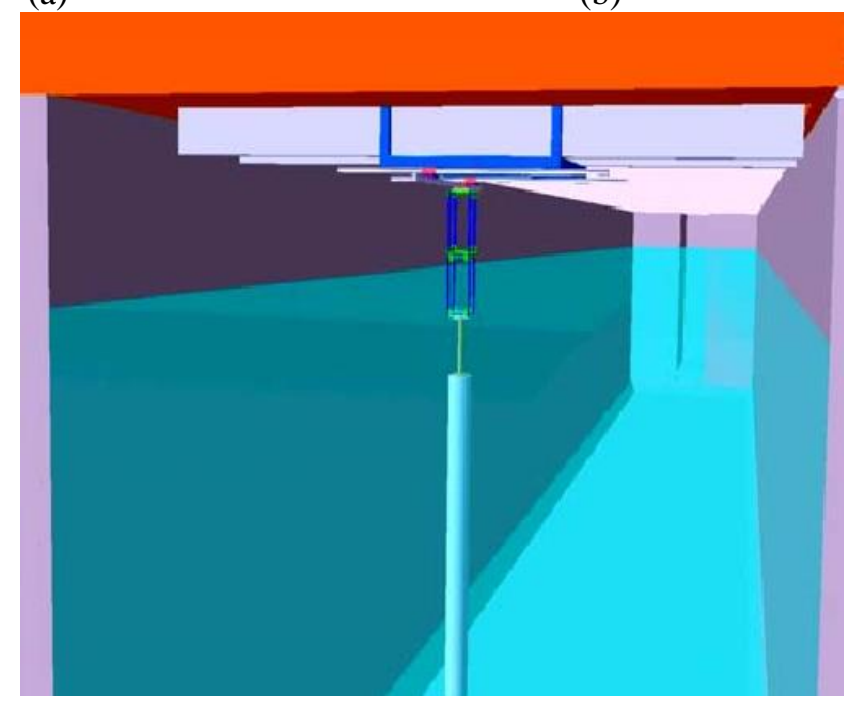

(c)

Fig. 1. Testing auxiliary device for two-degrees-of-freedom VIV of cylinders, (a) 2-D design diagram, (b) 3D view of the device model, and (c) installation diagram of the whole auxiliary device and a single cylinder.

As shown in Fig. 1 (a) and (b), the main body of auxiliary device could be divided into two parts: inline and cross-flow motion parts corresponding to Part 1 and Part 2 respectively, which are composed of three flats arranged horizontally and eight connecting rods with the same length. Deformation would not occur to the flats and connecting rods for their great stiffness. The connections between the flats and the rods are rotating bearings, and the rotating bearings of Part1 are perpendicular to those of Part 2, which can ensure the Part 1 and Part 2 are able to rotate in the in-line and cross-flow directions respectively. As a result, the bottom flat in Part 2 could satisfy the requirements of two-degrees-of-freedom vibration well. It should be noted that the flats in the auxiliary device won't rotate when the two-degrees-of-freedom motion occurs, 
which is due to the every four connecting rods between two flats are with the same length. That means the flats will only make rigid body vibrations.

The testing circular cylinders are made of UPVE pipes, with the diameter of $11 \mathrm{~cm}$, wall thickness of 5 $\mathrm{mm}$, and length of $1.0 \mathrm{~m}$, as shown in Fig 2 . The bottom and top parts of the cylinder models are sealed, and their insides are filled with foam material to avoid the water seepage and reduce the mass ratio parameter relating to easily observed large amplitudes. In addition, a steel rod with the diameter of $1 \mathrm{~cm}$ and length of $30 \mathrm{~cm}$ was installed on the top of each cylinder, which is to connect with the testing auxiliary device and keep the cylinder under the water.

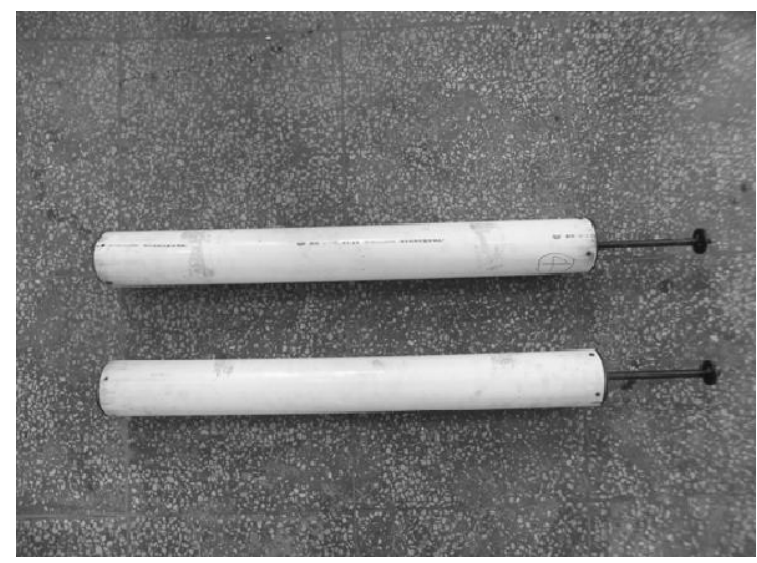

Fig. 2. Pictures of the testing cylinder models in this experiment.

The top plate of the testing auxiliary device is fixed on an auxiliary steel frame to keep the whole fitting parts stable. The auxiliary steel frame is a square frame structure rigidly fixed on the longitudinal beam of the carriage, which is seen as the base of the testing models.

It should be pointed out that the vibration of the cylinders involves the cross-flow and in-line motions as well as the vertical movement. Therefore, during the whole testing process, the cylinder models is completely submerged in the water, the testing auxiliary device is above the water and only the steel connecting rod moves through the water surface periodically. Considering the cylinder model and the bottom plate in Part 2 have been fixed stably, a three-component acceleration sensor is installed on that plate of the testing auxiliary device to measure the vibration signal of each cylinder, and the amplitude results are further processed on basis of the acceleration data.

Table 1 displays the key parameters of the upstream and downstream cylinders. The mass ratios of upstream and downstream cylinders are 1.048 and 1.049 respectively.

Table.1 Parameters of the cylinder models 


\begin{tabular}{ccccc}
\hline Item & Length & Diameter & Wet weight of cylinder & Weight of auxiliary device \\
\hline Upstream cylinder & $1 \mathrm{~m}$ & $0.11 \mathrm{~m}$ & $0.46 \mathrm{~kg}$ & $4.20 \mathrm{~kg}$ \\
Downstream cylinder & $1 \mathrm{~m}$ & $0.11 \mathrm{~m}$ & $0.47 \mathrm{~kg}$ & $4.18 \mathrm{~kg}$ \\
\hline
\end{tabular}

The two-degrees-of-freedom VIV experiments of an isolated cylinder and two staggered cylinders were carried out systematically under the condition of different center spacing ratios and flow velocities. The relevant pictures of the models and devices are shown in Fig. 3.

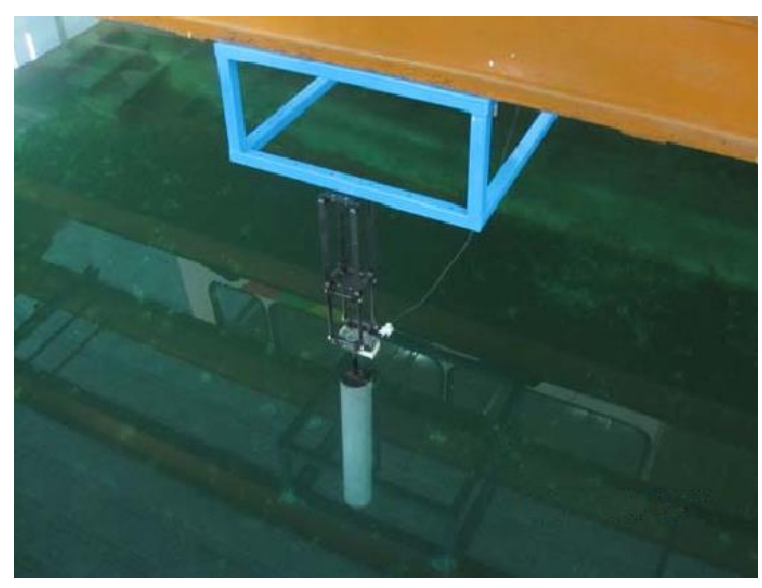

(a)

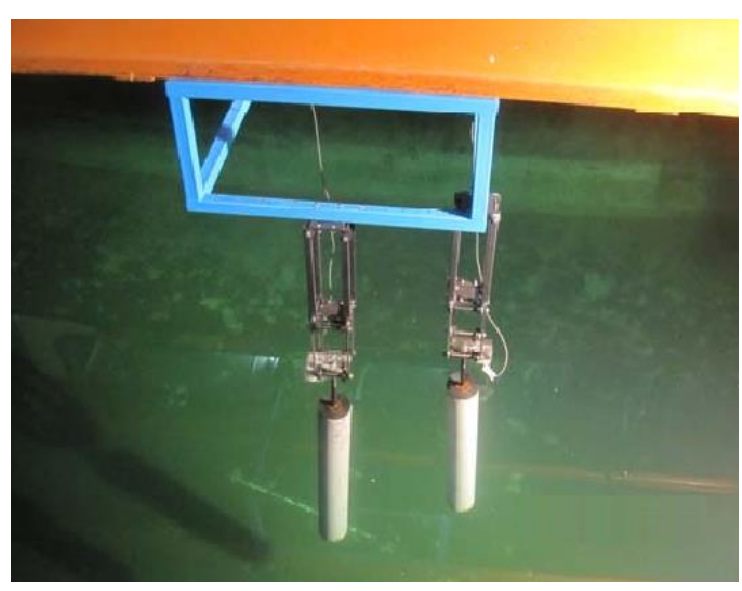

(b)

Fig. 3. Installation diagram of the two-degrees-of-freedom VIV experiments, (a) single cylinder, and (b) two staggered cylinders.

When the centerline of the two cylinders shows a certain angle with the flow direction, it is called the staggered arrangement. We define the in-line gap between the cross section centers of two cylinders as $L$, the cross-flow gap between those as $T$, and the ratio of $L$ or $T$ to the cylindrical diameter as in-line gap ratio $L / D$ or cross-flow gap ratio $T / D$, shown in Fig. 4 .

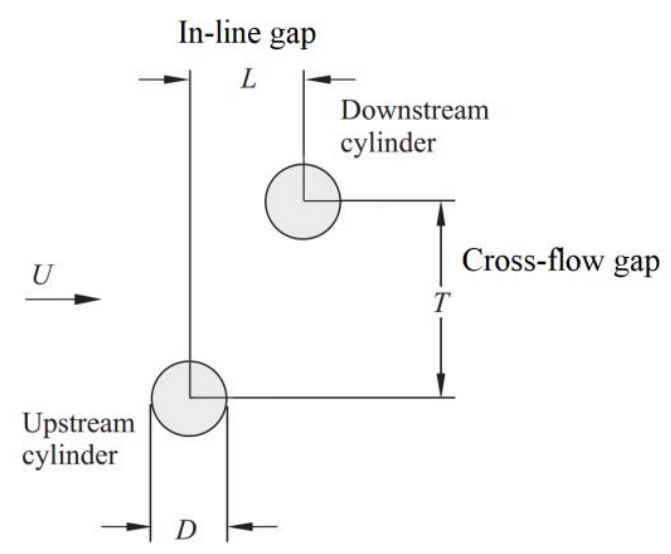

Fig. 4. Parameters in staggered arrangement of the two circular cylinders.

In order to investigate the effects of gap ratios on the two-degrees-of-freedom VIV response of a pair of 
staggered circular cylinders, we set the in-line gap ratio $L / D$ as 3.0, 3.6, 4.2 and 5.5, the cross-flow gap ratio $T / D$ as $0.7,1.1,1.5,1.9,2.3$ and 2.7 respectively. There are 24 different cases about the combination of inline and cross-flow gap ratios in the experiment of two staggered circular cylinders, and the case with $L / D=3.6$ and $T / D=0.7$ is first selected as a typical one to compare with that of a single, where the flow velocity ranges from $0.2 \mathrm{~m} / \mathrm{s}$ to $0.8 \mathrm{~m} / \mathrm{s}$ with an interval of $0.02 \mathrm{~m} / \mathrm{s}$. Then, comprehensively considering the experimental efficiency and accuracy, we set the flow velocity interval as $0.04 \mathrm{~m} / \mathrm{s}$ with the constant range of $0.2 \mathrm{~m} / \mathrm{s}$ to $0.8 \mathrm{~m} / \mathrm{s}$ in other cases. The experimental Reynolds number in all cases ranges from 22000 to 88000 .

\section{Results and discussion}

The dynamic responses of the models are described in terms of reduced amplitude $A / D$ versus reduced velocity $U_{r}$. Amplitude peaks were calculated employing the Hilbert transform, as described in Khalak and Williamson ${ }^{19}$. The reduced velocity $U_{r}=U / f_{n y} D$ ranges from 5.32 to 21.28 , covering the occurrence of several possible phenomena, where $U$ represents flow velocity, $D$ is the diameter of the cylinder and $f_{n y}$ is the crossflow natural frequency.

\subsection{Single circular cylinder}

Before a series of vibration tests, we need to obtain the natural frequencies and the damping ratios of the two cylinders by conducting the free decay tests in cross-flow and in-line directions. Fig. 5 illustrates the time-domain free decay curve and PSD results of the cross-flow acceleration of upstream cylinder.

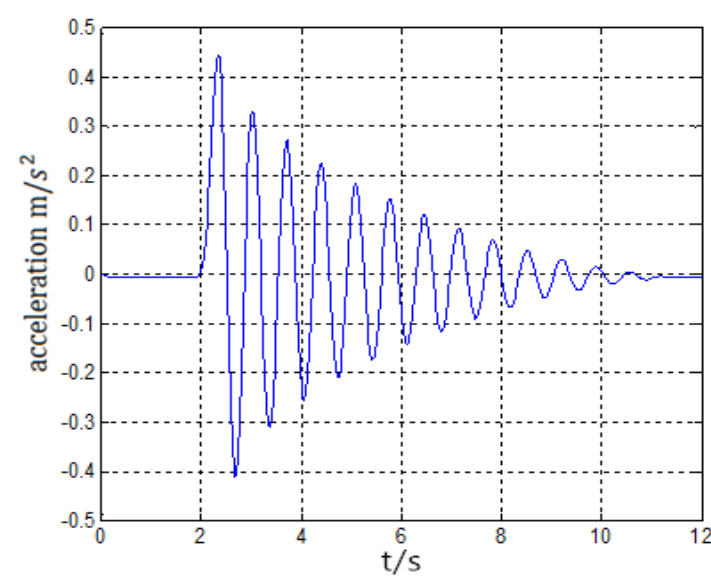

(a)

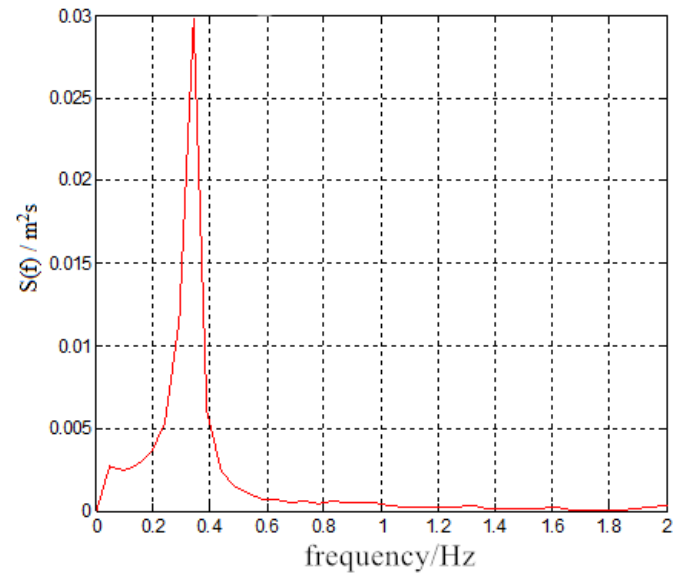

(b)

Fig. 5. Free decay results of cross-flow acceleration of upstream cylinder, (a) time-domain curve, and (b) PSD processing curve. 
By observing the time-domain curve and analyzing the power spectral density (PSD) processing curve in Fig. 5, the cross-flow natural frequency of upstream cylinder is determined to be $0.3418 \mathrm{~Hz}$. Similarly, the in-line natural frequency of upstream cylinder and the two-direction frequencies of downstream cylinder are also obtained by the same method.

The damping ratio of each single cylinder system can be calculated by the decay rate $\delta$ measured in the free decay model test, which is described by

$$
\delta=\ln \frac{y_{1}}{y_{2}}=\frac{2 \pi \xi}{\sqrt{1-\xi^{2}}}
$$

where $y_{1}$ and $y_{2}$ are two adjacent amplitudes on time-domain decay curve, $\xi$ is the damping ratio which represents the ratio of structural damping to critical damping ${ }^{20}$.

The relevant natural frequencies and damping ratios are given in Table 2.

Table.2 Natural frequencies and damping ratios of the two cylinders

\begin{tabular}{|c|c|c|c|}
\hline Item & Direction & Natural frequency $f_{n}(\mathrm{~Hz})$ & Damping ratio \\
\hline \multirow{2}{*}{ Upstream cylinder } & Cross-flow & 0.3418 & 0.0841 \\
\hline & In-line & 0.3456 & 0.0822 \\
\hline \multirow{2}{*}{ Downstream cylinder } & Cross-flow & 0.3427 & 0.0838 \\
\hline & In-line & 0.3449 & 0.0826 \\
\hline
\end{tabular}

Considering the high similarity of the parameters about the two cylinders, we take the upstream cylinder as the objective in the research on single cylinder. The case with a flow velocity of $0.4 \mathrm{~m} / \mathrm{s}$ is chosen as an example, and the cross-flow and in-line acceleration curves in time domain are shown in Fig. 6.
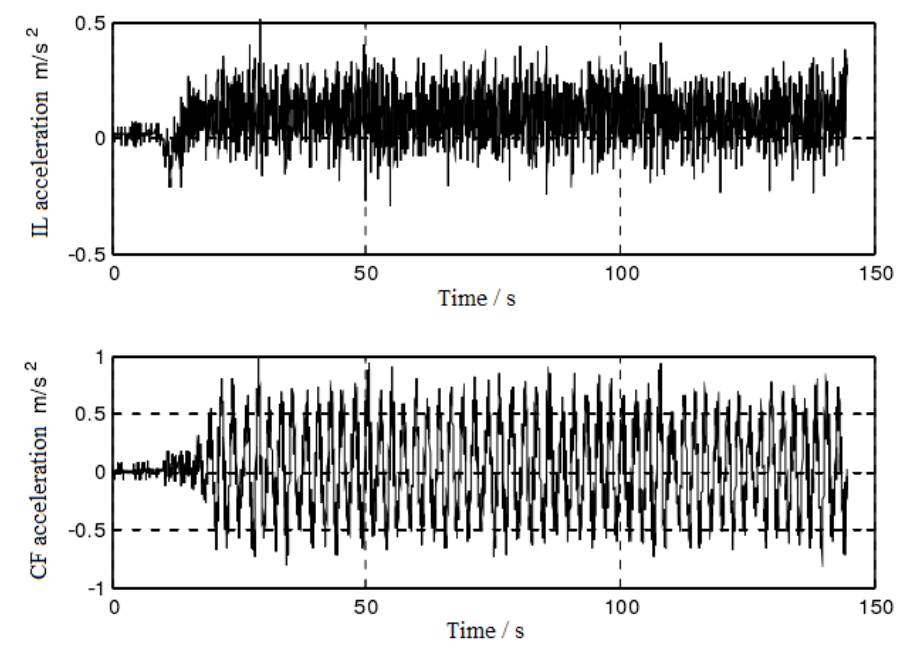

Fig. 6. Cross-flow and in-line vibration results of a single cylinder, where IL represents In-line direction and CF represents the cross-flow direction. 
It can be observed that the testing curves have poor smoothness and repeatability due to the existence of the interference like noise. Thus, the low-pass and high-pass filtering processes are preformed to solve that problem. Considering both the cross-flow and in-line natural frequencies are about $0.34 \mathrm{~Hz}$ and the harmonic frequency of cylindrical VIV above the fifth order has limited contribution to the response, the low-pass and high-pass frequencies are selected as $2 \mathrm{~Hz}$ and $0.1 \mathrm{~Hz}$. Then, the fast Fourier transform (FFT) is applied to obtain the PSD function of the vibration. The processed results over a period of time of cross-flow and inline vibrations are given in Fig. 7.

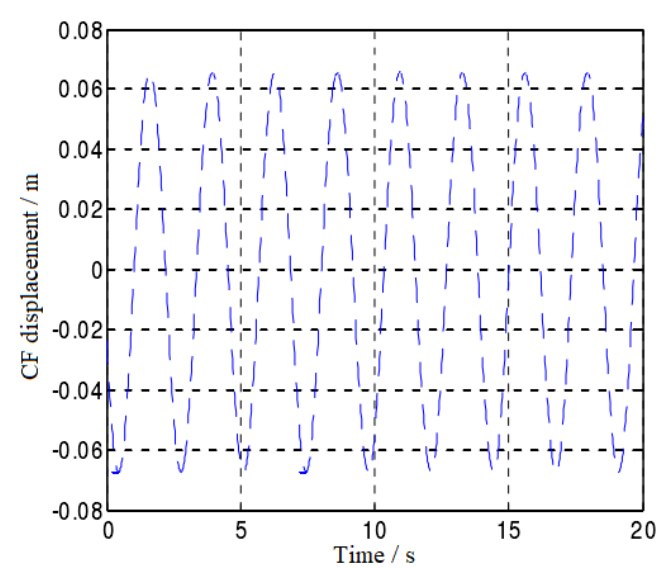

(a)

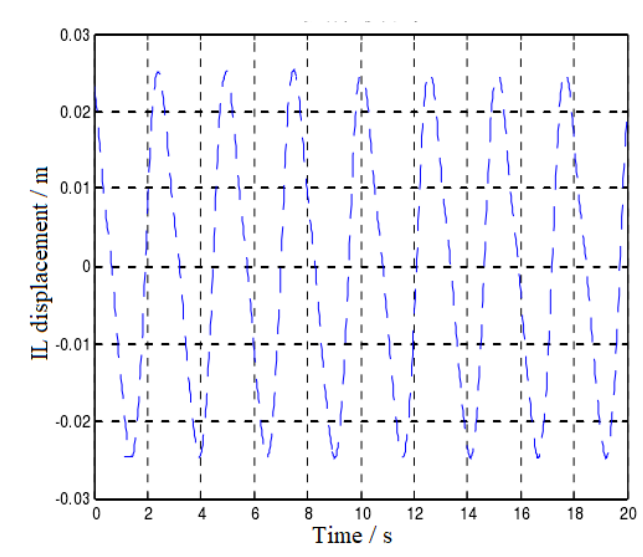

(c)

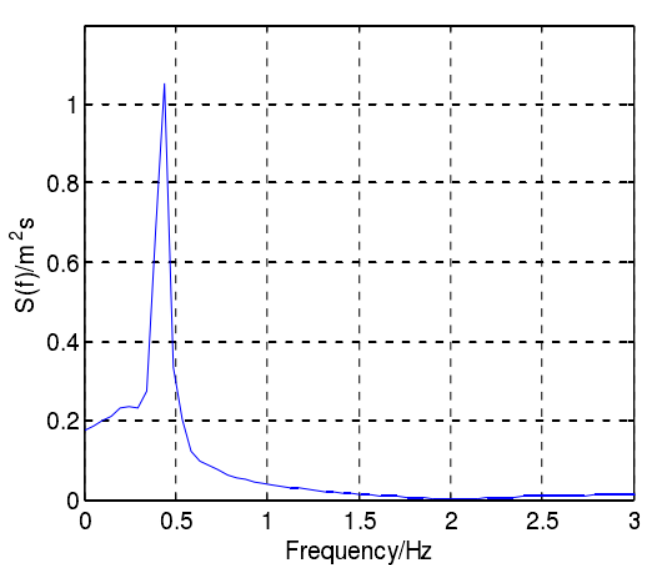

(b)

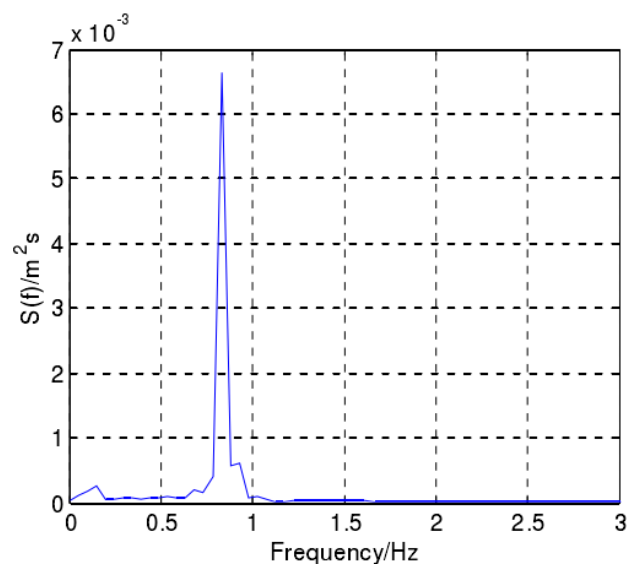

(d)

Fig. 7. The processed results of cross-flow and in-line vibrations of the single cylinder, (a) cross-flow displacement in time-domain, (b) PSD results of cross-flow vibration, (c) in-line displacement in timedomain, and (d) PSD of in-line vibration.

By observing the curves in Fig. 7, we can find that the single cylinder vibrates with the main frequency of $0.4395 \mathrm{~Hz}$ and the maximum amplitude of $0.0656 \mathrm{~m}$ in cross-flow direction, and with the main frequency 
of $0.879 \mathrm{~Hz}$ and the maximum amplitude of $0.0251 \mathrm{~m}$ in in-line direction. The frequency of in-line vibration is about twice of that of cross-flow vibration, which is consistent with VIV studies conducted by Jauvtis and Williamson ${ }^{18}$, Gao et al. ${ }^{21}$, and so on.

To further investigate the amplitude and frequency responses under various velocities, the vibration results of reduced amplitude $A / D$ and representative cross-flow dimensionless frequency $f_{y} / f_{n y}$ are presented in Fig.8. It should be noted that the amplitude $A$ is the root mean square value about the relevant time-domain curve. Meanwhile, the experimental results obtained by Kim et al. (2016) ${ }^{23}$ and Srinil et al. (2013) ${ }^{24}$ with the mass ratios of 1.1 and 1.4, cross-flow damping ratios of $4.6 \%$ and $1 \%$ and in-line damping ratios of $3.9 \%$ and $4.7 \%$ respectively are chosen as the comparison and shown in Fig.8.

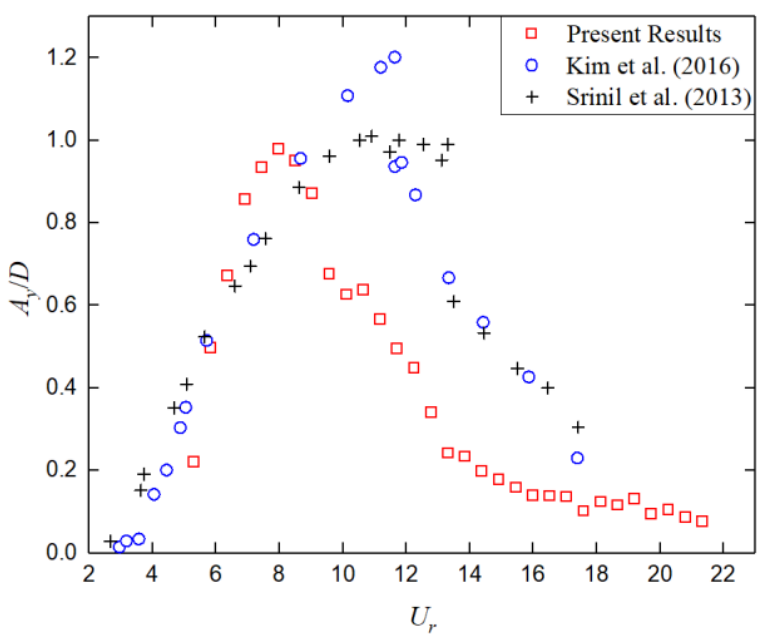

(a)

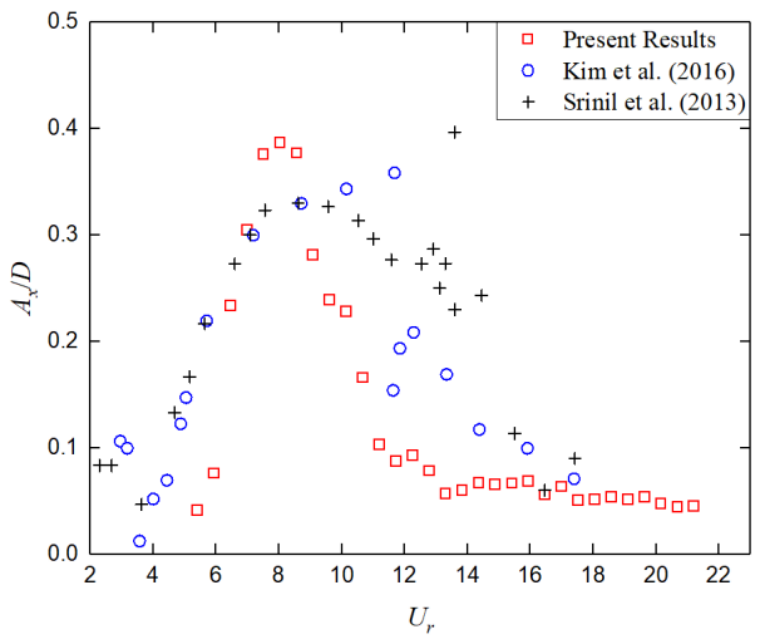

(b)

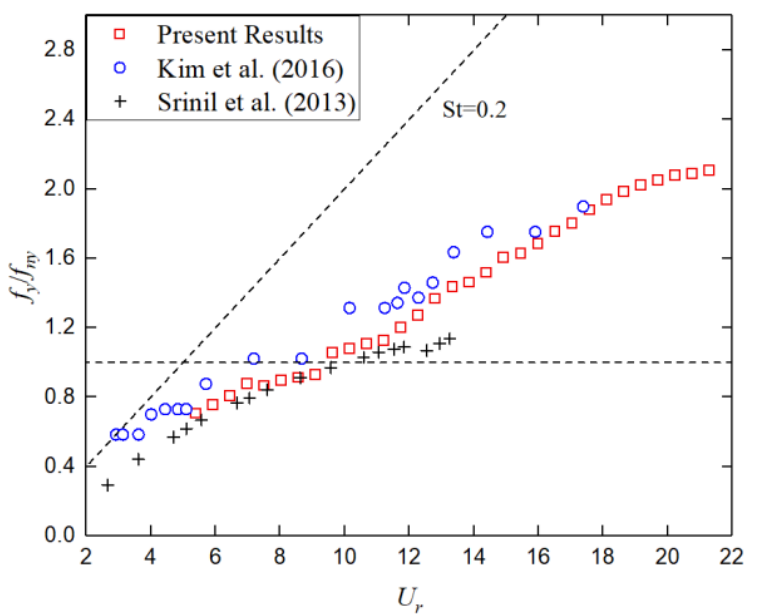

(c)

Fig. 8. The reduced amplitude and dimensionless frequency results under different reduced velocities, (a) cross-flow amplitudes, (b) in-line amplitudes, (c) cross-flow frequencies. 
It can be observed from the present results in Fig. 8 that the cross-flow and in-line amplitudes reach the maximum value of $0.98 \mathrm{D}$ and $0.39 \mathrm{D}$ respectively when the reduced velocity is near 8 , and the dimensionless cross-flow vibration frequency is near 0.9 corresponding to the lock-in region at this time. When the reduced velocity is larger than 8 , the cross-flow and in-line amplitudes of the cylinder decrease sharply, and the vibration is tending to jump out of the lock-in region, which is in accordance with the classical theory of cylindrical VIV. Although the maximum value and the width of lock-in interval of the present results show some differences with those obtained by Kim et al. and Srinil et al., which is possibly caused by the different damping ratios, the overall trends of both amplitude and frequency are basically consistent. The effect of the damping ratio can also be found by comparing the results of Kim et al. with those of Srinil et al. Taking the cross-flow amplitude as an example, we can observe that the reduced velocity corresponding to the decreasing point of Kim's results is smaller than that of Srinil's results due to the different cross-flow damping ratio. Thus, the damping ratio of more than $8 \%$ in this paper, inducing the smaller reduced velocities corresponding to the decreasing point, is the most possible reason for the difference among present, Kim's and Srinil's results. Generally, the present results can reasonably reflect the main features of the 2-DOF VIV of a cylinder, which verifies the feasibility of the test devices.

\subsection{Two staggered circular cylinders}

\subsubsection{Vibration responses of the two staggered circular cylinder $(L / D=3.6, T / D=0.7)$}

The staggered arrangement is an extension of a pair of adjacent cylinders to arbitrary flow direction, which may result in some more completed characteristics and newfangled phenomena. In this section, the case of $L / D=3.6$ and $T / D=0.7$ is selected as an object to investigate the important amplitude and frequency responses systematically.

The reduced cross-flow and in-line amplitudes and dimensionless cross-flow frequencies varying with the reduced velocity of the upstream, downstream and isolated cylinders, are shown Fig. 9. 


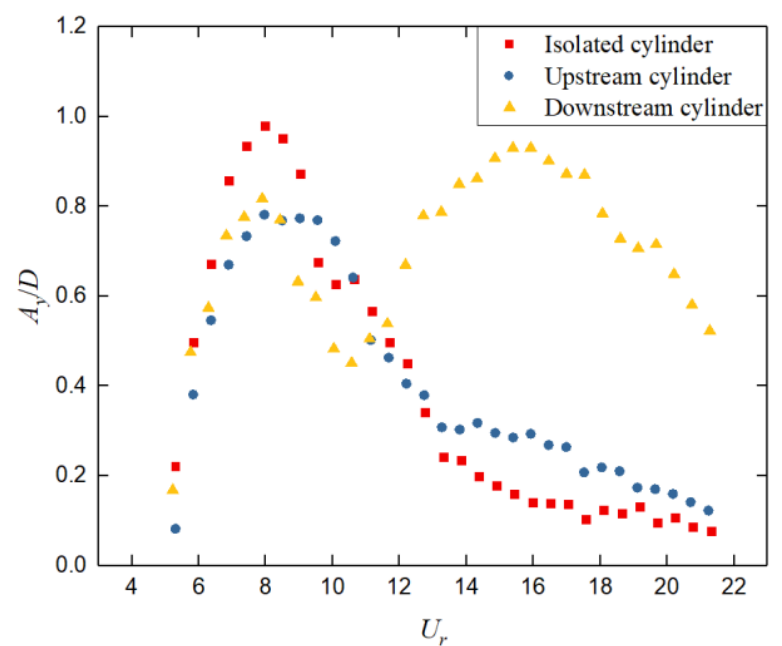

(a)

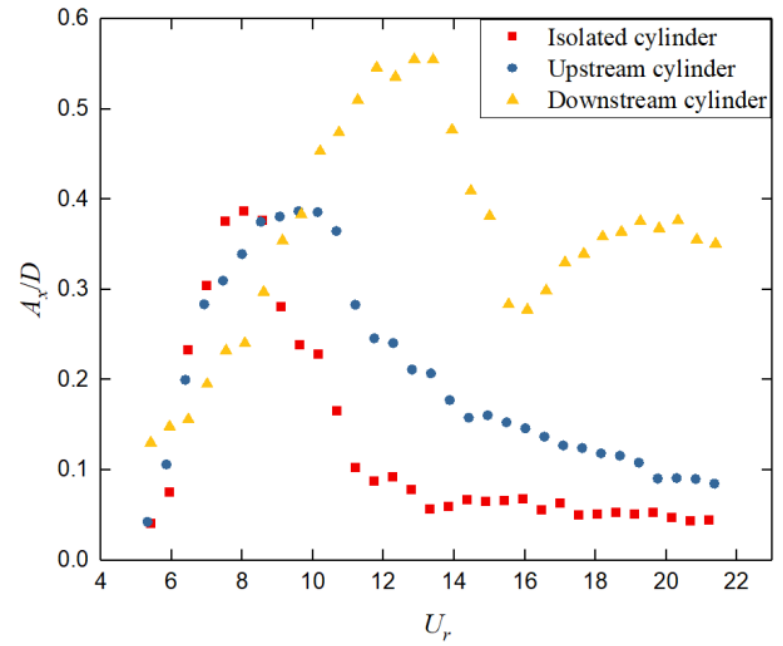

(b)

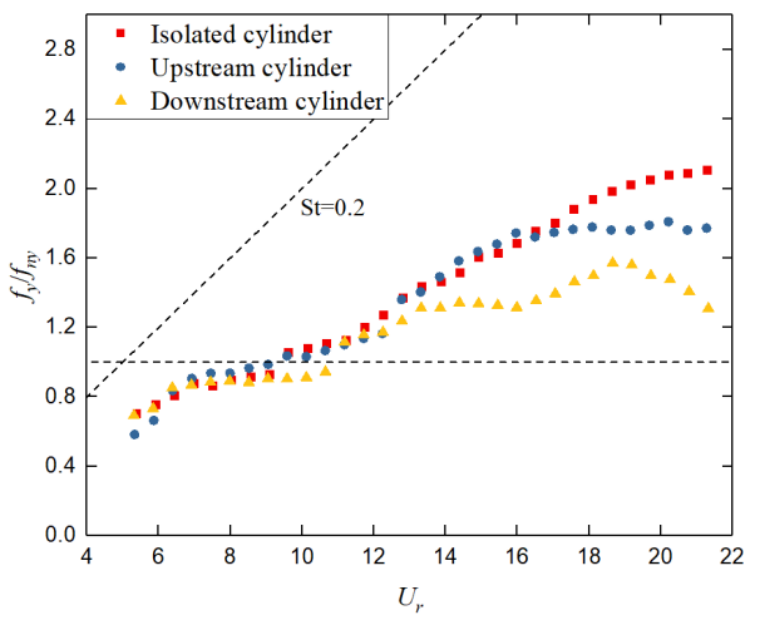

(c)

Fig. 9. Comparison of the amplitude responses of two staggered circular cylinders and a single circular cylinder, (a) cross-flow amplitudes, (b) in-line amplitudes, (c) cross-flow frequencies.

As shown in Fig. 9 (a), the cross-flow amplitudes of the upstream cylinder have the similar trend with the isolated single cylinder. With the increasing of reduced velocity, the cross-flow vibration amplitudes of the upstream cylinder and single isolated cylinder increase firstly, but then decrease sharply when reduced velocity is larger than about 8 . When the reduced velocity reaches 20 , the cross-flow vibration amplitudes of the upstream cylinder and the isolated single cylinder are very small. At the same time, it can be found that the maximum cross-flow amplitude of the upstream cylinder is smaller than that of the isolated cylinder, which may be due to that the existence of downstream cylinder disturbs the wake vortex behind the upstream cylinder and further reduces the associated fluctuating lift. But the amplitude of the upstream cylinder at higher reduced velocity $\left(U_{r}>13\right)$ is larger to some extent, which may be owing to the vortex enhancement 
caused by high-amplitude vibration of downstream cylinder.

It is obvious that there is a large difference about the cross-flow amplitudes between the downstream cylinder and the upstream cylinder and isolated single cylinder. As described in above paragraphs, the total variation trend about the cross-flow amplitudes of the downstream cylinder presents two peaks, and the amplitudes near the second peak are still large, which is different from the "double peaks" phenomenon about side-by-side arrangement of two cylinders whose amplitudes are obviously smaller near the second peak. In addition, when reduced velocity is larger than the value corresponding to the second peak of downstream cylinder in staggered arrangement, the amplitude value starts to decrease, which also differs from the wake galloping phenomenon in tandem cases $^{22}$. In general, the special "two peaks" phenomenon of staggered cylinders, showing the two maximum amplitude values at different reduced velocities and the large but limited amplitude responses at high reduced velocities, is a combination of the above two phenomena.

For the in-line amplitude results illustrated in Fig. 9 (b), similar behaviors are observed for the upstream and downstream cylinders. However, some slight differences still exist between the cross-flow and in-line amplitudes. The strengthening effect of downstream cylinder on the in-line amplitude of upstream cylinder at high reduced velocities is more obvious. For the downstream cylinder, there is also an obvious "double peaks" phenomenon appearing on the in-line amplitude response, but the first peak is larger than the second one. And the reduced velocity of maximum amplitude of downstream cylinder is obviously lagging behind those of upstream cylinder and the isolated single cylinder. The possible reason may be that the vortex produced by the upstream cylinder would move further under higher flow velocity and thus interact with the vortex generated by the downstream cylinder, which enhance the fluctuating drag acting on the downstream cylinder.

In addition, we can find in Fig. 9 (c) that the dimensionless cross-flow frequencies of the upstream, downstream and isolated cylinders share the similar trend when reduced velocity is smaller than about 13 . The three kinds of results all experience a lock-in region where the dimensionless frequencies ranges from about 0.9 to 1.1 and the reduced velocity is about $U_{r}=7 \sim 11$. But when $U_{r}>13$, the downstream cylinder shows another lock-in region until $U_{r} \approx 16$ where the frequencies are near 1.3, corresponding to the second peak in the amplitude responses. In addition, because of the more serious disturbance and interference of flow at high reduced velocities, the cross-flow vibration frequency of downstream cylinder decreases with the increase of 
the reduced velocity, which is completely different from the Strouhal principle. In terms of the upstream cylinder, the dimensionless cross-flow frequency of the upstream cylinder arrives at the maximum of 1.81 when the reduced velocity is about 20 . When the reduced velocity continues to increase, the cross-flow frequency tends to be stable, which is different from that of the isolated cylinder.

\subsubsection{Effect of cross-flow and in-line gap ratios on amplitude responses}

Through the above analyses, we can find that the upstream cylinder has some significant effects on the downstream cylinder in staggered arrangement, especially for amplitude characteristics. The gap distance between the cylinders has a major effect on the movement and development of the wake vortex, which will further affect the VIV characteristics of the cylinders. Considering the primacy and mutability of vibrating amplitude in VIV predictions, the effect of in-line and cross-flow gap ratios on the amplitude responses of two staggered cylinders is investigated systematically. It should be noted that the frequency variation of the upstream cylinder is similar with that of an isolated cylinder except for the limited difference in high-velocity region. Therefore, the analyses on frequency are only conducted for the downstream cylinder in this section.

By adopting the control variable method, we first explore the influence of cross-flow gap ratio on vibration amplitude responses. As a result, the in-line gap ratio $L / D$ is set as 3.6 constantly, and the crossflow gap ratio $T / D$ is selected as $0.7,1.1,1.5,1.9,2.3$, and 2.7 respectively in the experiment, as described in Section 2. It should be noted that the case of $T / D=0$ is not discussed here because that is defined as the tandem arrangement whose response characteristics may be consulted in some current papers published by Korkischko and Meneghini ${ }^{14}$, Assi ${ }^{3}$, Song et al. ${ }^{22}$ and other scholars. The measuring cross-flow and in-line amplitude results about upstream and downstream cylinders with different cross-flow gap ratios are shown in Fig. 10. 


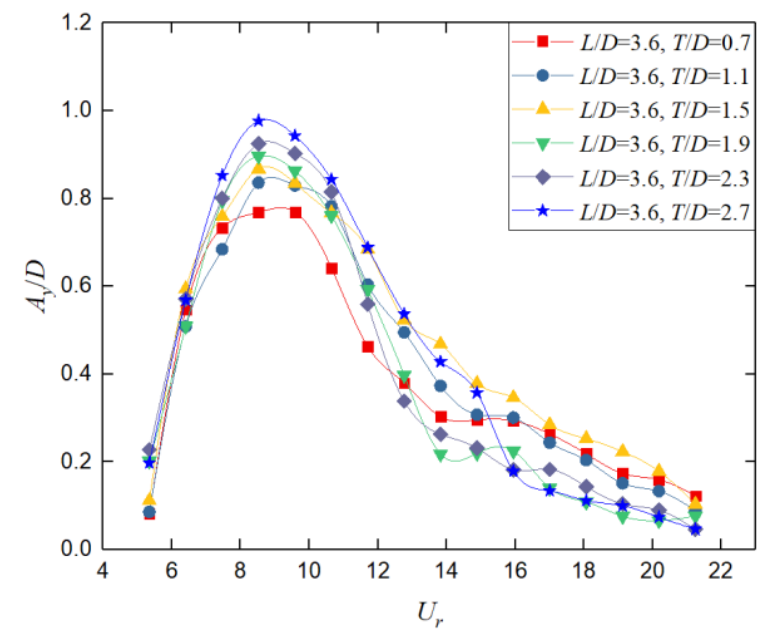

(a)

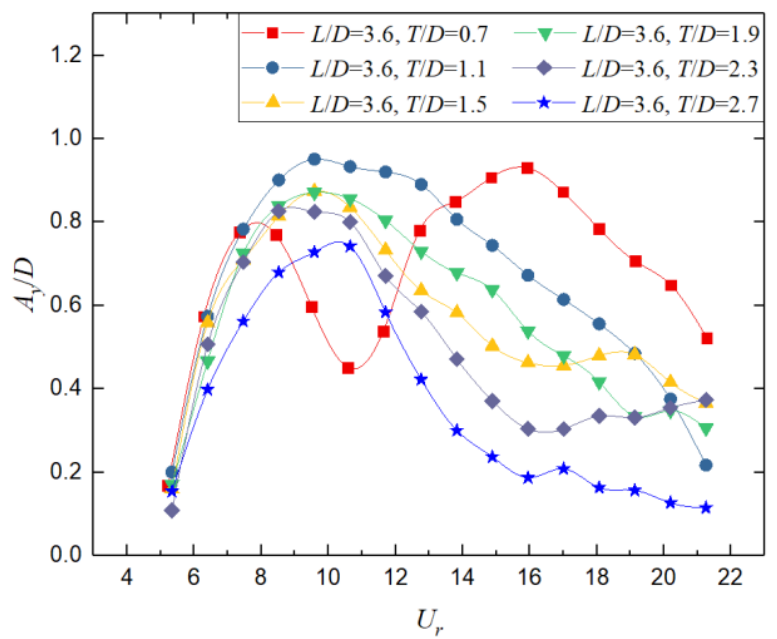

(c)

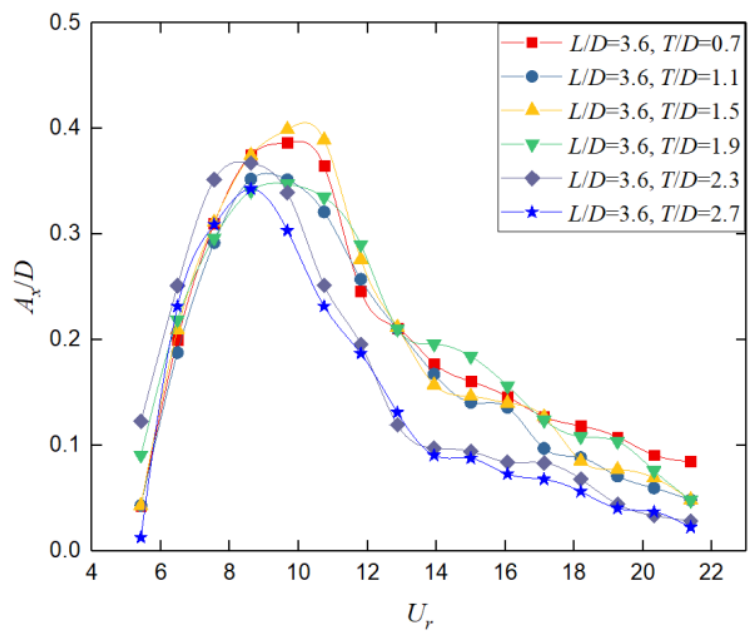

(b)

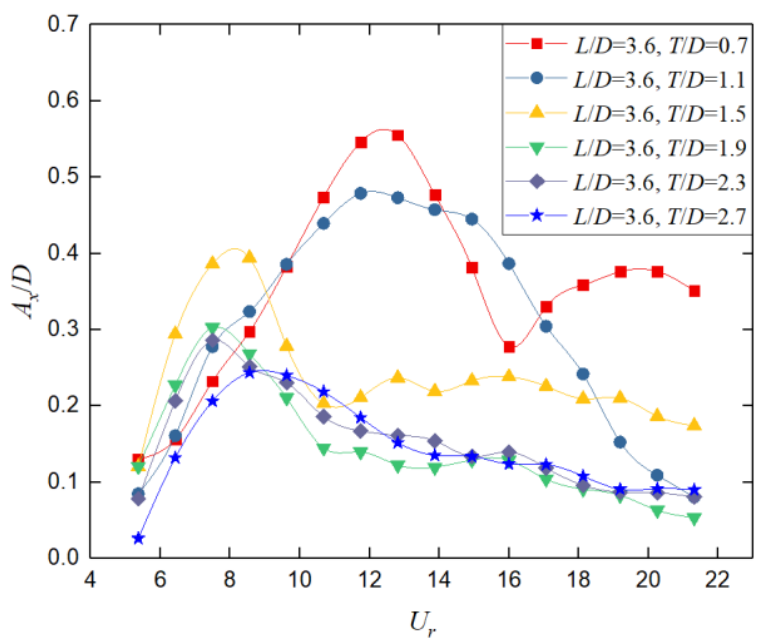

(d)

Fig. 10. Comparison of the reduced amplitude results of the staggered cylinders with $L / D=3.6$ and $T / D=0.7$,

$1.1,1.5,1.9,2.3$, and 2.7 under different reduced velocities, (a) cross-flow amplitudes of the upstream cylinder, (b) in-line amplitudes of the upstream cylinder, (c) cross-flow amplitudes of the downstream cylinder, (b) in-line amplitudes of the downstream cylinder.

As shown in Fig. 10 (a), when the reduced velocity is about 7 to 9, the cross-flow vibration amplitude of the upstream cylinder reaches the maximum value corresponding to the "lock-in" region, which is basically consistent with the variation trend of the isolated-cylinder amplitudes described in above sections. When the in-line gap ratio $L / D$ is kept as 3.6, the maximum value of the cross-flow amplitudes of the upstream cylinder gradually increases with the increasing of the cross-flow gap ratio $T / D$, indicating that the amplitude suppression of upstream cylinder induced by the downstream cylinder (see the comparison between the staggered cylinders and the isolated cylinder described below Fig. 9) is gradually weakened by the increasing 
cross-flow gap ratio. When the reduced velocity is larger than about 15 , the amplitude responses in the cases of $T / D=0.7,1.1,1.5$ and 1.9 are relatively larger than those in the other cases, and a phenomenon of mutual cross exists due to the complexity of flow with high Reynolds number. For the in-line amplitudes of upstream cylinder in Fig. 10 (b), some similar characteristics are found. All off six cases have the similar variation trend when the reduced velocity is increasing. The main effects of cross-flow gap ratio on in-line amplitudes of upstream cylinder are reflected in the width of large-amplitude region and the value of amplitude at high reduced velocities.

Unlike the upstream cylinder, the downstream cylinder is more sensitive to the variation of cross-flow gap ratio. By observing the cross-flow results of downstream cylinder in Fig. 10 (c), we can find that the width of large-amplitude region is increasing with the decrease of cross-flow gap ratio, and a special "double peaks" phenomenon even occurs when $T / D=0.7$, indicating the strengthen effect of upstream cylinder on the amplitude responses of downstream cylinder is enlarged by the decreasing $T / D$. The possible reason may be that the wake vortexes generated by upstream cylinder are closer to the downstream cylinder with the decreasing of $T / D$ and thus easily interact with original vortexes of downstream cylinder, further enhancing the power of the vortex region near downstream cylinder and increasing its response. In addition, because of the vortex strengthening of downstream cylinder, both the maximum amplitude and the overall amplitudes at high reduced velocities are larger in the cases with smaller $T / D$. For the in-line amplitudes of downstream cylinder in Fig. 10 (d), the effects of smaller cross-flow gap ratio are even more obvious and significant. When the $\mathrm{L} / \mathrm{D}=3.6$ and the $T / D=1.9,2.3$ and 2.7 , the variation trend of vibration amplitude is similar to that of the isolated single cylinder. When $T / D=1.5,1.1$ and 0.7 , the trend of amplitude with the increasing of reduced velocity is gradually changing to some extent. The enlargement of the amplitude at high reduced velocities, the obvious change of the value of reduced velocity corresponding to the maximum amplitude and the special double peaks successively appear when the $T / D$ is constantly decreasing, indicating the characteristics dominated by tandem arrangement is becoming more and more obvious because the smaller $T / D$ means more closer relationship with tandem arrangement. The flow in front of the downstream cylinder is blocked by the upstream one to some extent when the $T / D$ is small enough like in tandem arrangement, which means only larger flow velocity can provide the enough energy for resonance. So the reduced velocity corresponding to the maximum amplitude has been enlarged when $T / D=1.5,1.1$ and 0.7 . When the flow 
velocity continuously increases, the vortexes behind upstream cylinder may move to the region around the downstream cylinder fast, further strengthening the vortex energy for downstream cylinder and inducing the large amplitude. There are four different variation trends in the in-line amplitude results. Generally, both the cross-flow and the in-line amplitude responses of the downstream cylinder change greatly under different cross-flow gap ratios, and further researches and discussions will be conducted to explore the exact reasons for the concrete transition mechanism.

The above analyses of the effects of $T / D$ on the amplitude responses of the staggered cylinders are limited in the condition of $L / D=3.6$. Considering the more obvious and significant reaction of downstream cylinder and the larger-response cross-flow amplitude, we further take the cross-flow vibration amplitude and frequency of the downstream cylinder as the object to investigate the effects of other combinations of $T / D$ and $L / D$ in a short amount of space. The measuring cross-flow amplitude and frequency results about the downstream cylinder with the in-line gap ratio $L / D=3.0,3.6,4.2$ and 5.5 and cross-flow gap ratio $T / D=0.7$, $1.1,1.5,1.9,2.3$, and 2.7 in different reduced velocities are shown in Fig. 11. Similarly, the case of $L / D=0$ is not considered due to that is defined as side-by-side arrangement.

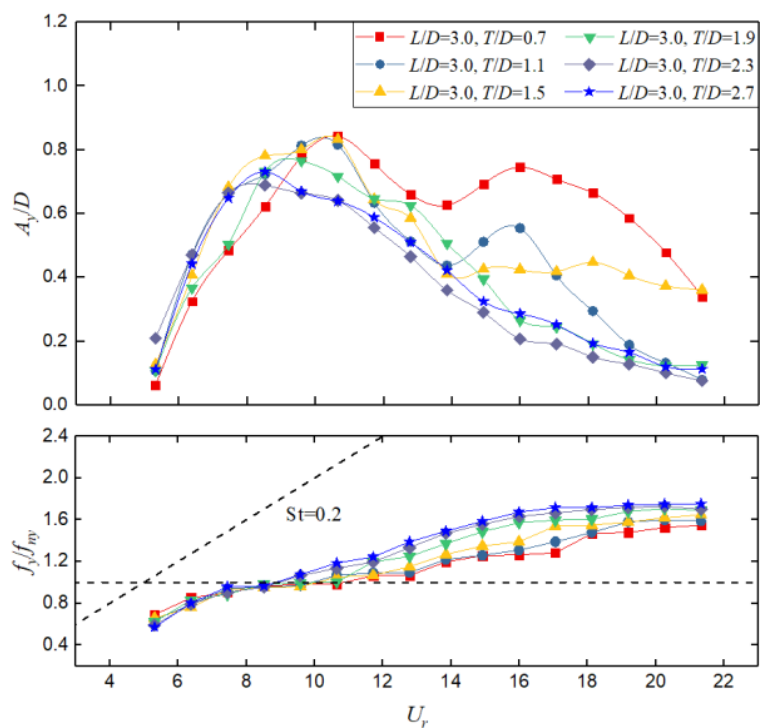

(a)

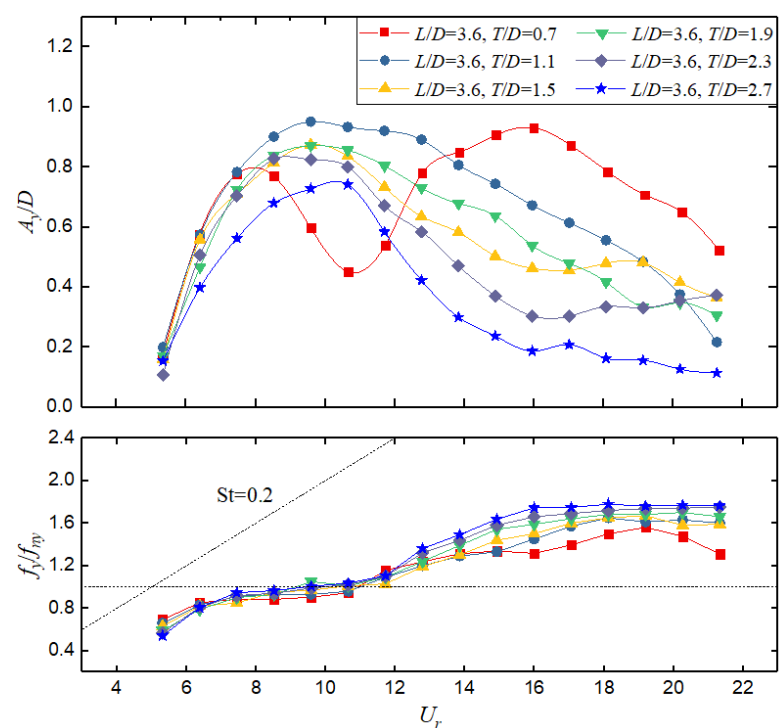

(b) 

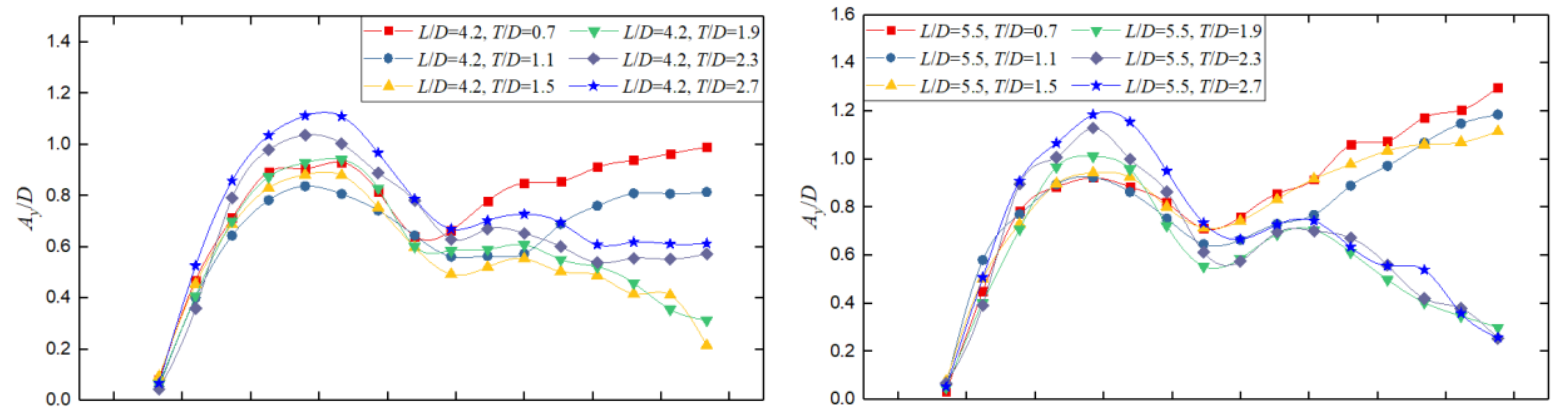

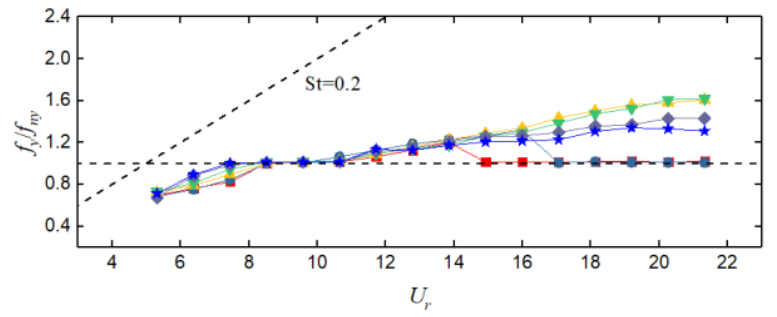

(c)

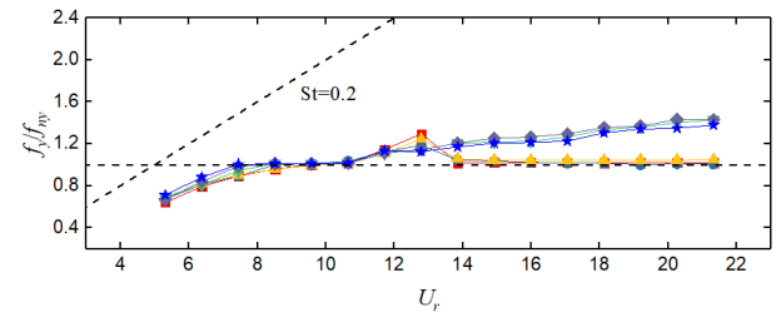

(d)

Fig. 11. Comparison of the reduced cross-flow amplitude and frequency results of the downstream cylinder with different combinations of $L / D$ and $T / D$ under different reduced velocities, (a) $L / D=3.0$ and $T / D=0.7,1.1$, $1.5,1.9,2.3$, and 2.7 , (b) $L / D=3.6$ and $T / D=0.7,1.1,1.5,1.9,2.3$, and 2.7 , (c) $L / D=4.2$ and $T / D=0.7,1.1,1.5$, 1.9, 2.3, and 2.7, and (d) $L / D=5.5$ and $T / D=0.7,1.1,1.5,1.9,2.3$, and 2.7 .

For the results in Fig. 11 (a), the width of high-amplitude interval does not change until $T / D \leq 1.1$, and the effect of $T / D$ mainly appears in high-velocity region. The vortexes produced by upstream cylinder have limited effect on the downstream cylinder when $T / D$ is larger and $L / D=3.0$ possibly because the deflection of the line connecting their central points is so large that the downstream cylinder is out of the upstream vortex region. But when $T / D$ is small enough, the upstream vortexes are able to reach the area around the downstream cylinder, causing the vortex strengthening described in above analysis, which is especially obvious in high-velocity region. As for the frequency results, after an overlap in low-velocity region, the frequency curves separate with each other, where the results with lower $T / D$ are generally smaller than those with higher $T / D$ possibly because the amplitudes of the former is far larger than the latter under the same velocity and the system need the different frequencies to balance the total energy.

The amplitude results in Fig. 11 (b) have been analyzed in above parts. In term of the frequency results, similar phenomena with those in Fig. 11 (a) have been found, but their lock-in intervals are larger than those in Fig. 11 (a), corresponding to the different curve shapes about amplitude. The possible reason is that the reasonable increase of $L / D$ makes the downstream cylinder begin to step into the upstream vortex region as described above. 
The amplitude responses in Fig. 11 (c) appear an important phenomenon called wake-induced vibration (WIV), which is usually observed in the tandem arrangement of two cylinders. When $T / D=0.7$ and 1.1 , the amplitudes at high reduced velocities are continuously increasing instead of reducing to small values in classic VIV. This may be caused by the similar arrangement with tandem as the $L / D$ is relatively far larger than $T / D$. Under the high-velocity condition, some vortexes produced by upstream cylinder are possible to be flowed away and mix with other vortexes behind the downstream cylinder, thus generating larger lift. Accordingly, the downstream cylinder is easy to reach resonance and the vibration frequency is locked in the natural frequency of the cylinder, as shown in Fig. 11 (c). Similarly, the results in Fig. 11 (d) show the WIV phenomenon in some cases. This phenomenon is even more obvious in those results of $T / D=0.7,1.1$ and 1.5 as the further increasing of $L / D$ to 5.5 grows the area of upstream vortexes and facilitate their movement to the downstream vortex region. The frequencies also show the corresponding variations where the double lock-in regions appear in the cases of $T / D=0.7,1.1$ and 1.5.

The cross-flow amplitudes of the downstream cylinder with different combinations of $L / D$ and $T / D$ all reach the first maximum values at the reduced velocity of approximately 8-10. With the increasing of in-line gap ratio $L / D$, the overall values of cross-flow amplitude gradually increase in both the high-amplitude and the high-reduced-velocity regions. When $L / D=3.0,3.6,4.2$ and 5.5, the cross-flow amplitude responses show great differences in different cases. When $L / D=3.0$ and 3.6, the variation trend of the cross-flow amplitude transforms from the "single peak" to "double peaks" with the decreasing of $T / D$, which is similar with the case of a pair of side-by-side cylinders to some extent, indicating the narrowing of $T / D$ strengthens the transverse vortex interaction between the two cylinders. But when $L / D=4.2$ and 5.5 , the variation trend changes from the "double peaks" to the phenomenon of wake galloping, namely WIV which usually appears in the case of tandem cylinders. The possible reason for the different effects of $T / D$ in the cases of different $L / D$ s is that the elongation of in-line gaps makes the staggered arrangement more similar with the tandem arrangement which will significantly change the flow around the downstream cylinder to different modes and further cause the WIV. And when the WIV happens, the vibration frequency will be locked around the natural frequency, indicating the inference of upstream vortexes induces the second resonance of the downstream cylinder.

In general, the cross-flow response characteristics of the downstream cylinder in staggered arrangement 
are the combination of those of the cylinder in isolated, side-by-side and tandem arrangements, and the dominated characteristics of the downstream cylinder depend on the arranged shape of the two cylinders, which is mainly affected by the cross-flow and in-line gaps.

\section{Conclusions}

In this paper, a set of testing devices for the VIV of two circular cylinders with two degrees of freedom has been designed, and then a series of VIV experiments of an isolated and two staggered cylinders are carried out. After the systematic analyses and specific comparisons of the response characteristics of the isolated and staggered cylinders under different conditions, the following conclusions are summarized:

(1) The results for an isolated cylinder are found to be in basic accordance with the regular characteristics of two-degrees-of-freedom VIV in currently published literatures. Consequently, the results are satisfactory to validate the experimental set-up and further provide reference for relevant numerical simulations.

(2) When $L / D=3.6$ and $T / D=0.7$, the amplitude and frequency differences between the upstream cylinder in staggered arrangement and the isolated cylinder are relatively smaller. But a special "double-peaks" phenomenon appears in both the cross-flow and in-line amplitudes of the downstream cylinder, which has not been found in the isolated-cylinder VIV studies. The possible reason for that is the wake of upstream cylinder also has an effect on the fluctuating lift acting on the downstream cylinder, and interference of the flow between the two cylinders may change the vortex modes of the downstream cylinder, which need further investigations on the fluid forces and vortex shedding mode of the two-degrees-of-freedom VIV of the staggered cylinders.

(3) Under the condition of $L / D=3.6$, the variation of $T / D$ has a limited effect on the amplitude responses of upstream cylinder but a significant impact on those of downstream cylinder. The widths of high-amplitude region of downstream cylinder in two directions are enlarged with decreasing of $T / D$, and even a totally different "double peaks" phenomenon of the amplitudes occurs at $T / D=0.7$.

(4) The variation of $L / D$ will change the effects of $T / D$ on the cross-flow amplitude characteristics of the downstream cylinder in staggered arrangement. When $L / D$ is smaller, namely $L / D=3.0$ and 3.6 in this experiment, the decreasing of $T / D$ promotes the transition of amplitude trend from the "single peak" to "double peaks". When $L / D$ is larger, namely $L / D=4.2$ and 5.5 in this experiment, the narrowing of $T / D$ generates a phenomenon of wake galloping with high amplitudes, namely "wake induced vibration", which 
may cause some serious damages for the real structures in engineering. Therefore, we'd better keep the arrangement of some engineering structures related to VIV, such as a group of marine risers or buoyancy cans, away from the above dangerous gap ranges.

\section{Acknowledgements}

The work presented here is supported by the Ph.D. Student Research and Innovation Fund of the Fundamental Research Funds for the Central Universities [grant number HEUGIP201803]; the National Natural Science Foundation of China [grant number 51879047], [grant number 51890915]; the National Science and Technology Major Project [grant number 2016ZX05028006-002]; and the Natural Science Foundation of Heilongjiang Province of China [grant number E2017029].

\section{References}

1. Kang Z, Zhang C, Chang R. A higher-order nonlinear oscillator model for coupled cross-flow and inline VIV of a circular cylinder. Ships \& Offshore Structures 2018; 13(5): 488-503.

2. Yun G, Zhi Z, Li Z, et al. Vortex-induced vibrations and waves of a long circular cylinder predicted using a wake oscillator model. Ocean Engineering 2018; 156: 294-305.

3. Assi GRS. Wake-induced vibration of tandem and staggered cylinders with two degrees of freedom. Journal of Fluids \& Structures 2014; 50: 340-357.

4. Prasanth TK, Mittal S. Vortex-induced vibration of two circular cylinders at low Reynolds number. Journal of Fluids \& Structures 2009; 25(4): 731-741.

5. Carmo BS, Meneghini JR, Sherwin SJ. Secondary instabilities in the flow around two circular cylinders in tandem. Journal of Fluid Mechanics 2010; 644(4): 395-431.

6. Derakhshandeh JF, Arjomandi M, Dally B, et al. The effect of arrangement of two circular cylinders on the maximum efficiency of Vortex-Induced Vibration power using a Scale-Adaptive Simulation model. Journal of Fluids \& Structures 2014; 49(8): 654-666.

7. Chen W, Ji C, Xu W, et al. Response and wake patterns of two side-by-side elastically supported circular cylinders in uniform laminar cross-flow. Journal of Fluids \& Structures 2015; 55: 218-236.

8. Zhao M. Flow induced vibration of two rigidly coupled circular cylinders in tandem and side-by-side arrangements at a low Reynolds number of 150. Physics of Fluids 2013; 25(12): 355-381. 
9. Tu J, Zhou D, Bao Y, et al. Flow-induced vibrations of two circular cylinders in tandem with shear flow at low Reynolds number. Journal of Fluids \& Structures 2015; 59: 224-251.

10. Mysa RC, Kaboudian A, Jaiman RK. On the origin of wake-induced vibration in two tandem circular cylinders at low Reynolds number. Journal of Fluids \& Structures 2016; 61: 76-98.

11. Hover FS, Triantafyllou MS. Galloping response of a cylinder with upstream wake interference. Journal of Fluids \& Structures 2001; 15: 503-512.

12. Allen DW, Henning, DL. Vortex-induced vibration current tank tests of two equal-diameter cylinders in tandem. Journal of Fluids \& Structures 2003; 17: 767-781.

13. Assi GRS, Meneghini JR, Aranha JAP, et al. Experimental investigation of flow-induced vibration interference between two circular cylinders. Journal of Fluids \& Structures 2006; 22(6): 819-827.

14. Korkischko I, Meneghini JR. Experimental investigation of flow-induced vibration on isolated and tandem circular cylinders fitted with strakes. Journal of Fluids \& Structures 2010; 26(4):611-625.

15. Huang S, Sworn A. Some Observations of Two Interfering VIV Circular Cylinders of Unequal Diameters in Tandem. Journal of Hydrodynamics 2011; 23(5): 535-543.

16. Huera-Huarte FJ, Gharib M. Flow-induced vibrations of a side-by-side arrangement of two flexible circular cylinders. Journal of Fluids \& Structures 2011; 27(3): 354-366.

17. Huang S, Herfjord K. Experimental investigation of the forces and motion responses of two interfering VIV circular cylinders at various tandem and staggered positions. Applied Ocean Research 2013; 43(43): 264-273.

18. Jauvtis N, Williamson CHK. The effect of two degrees of freedom on vortex-induced vibration at low mass and damping. Journal of Fluid Mechanics 2004; 509: 23-62.

19. Khalak A, Williamson CHK. Motions, forces and mode transitions in vortex-induced vibrations at low mass-damping. Journal of Fluids and Structures 1999; 13: 813-851.

20. Kang Z, Zhang C, Ma G, et al. A numerical investigation of two-degree-of-freedom VIV of a circular cylinder using the modified turbulence model. Ocean Engineering 2018; 155: 211-226.

21. Gao Y, Zong Z, L Zou, et al. Effect of surface roughness on vortex-induced vibration response of a circular cylinder. Ships \& Offshore Structures 2017; 13(1): 28-42.

22. Song Z, Duan M, Gu J. Hydrodynamics and vortex shedding characteristics of two tandem cylinders of 
different diameters in steady flow. Ships \& Offshore Structures 2015, 12(1): 86-99.

23. Shin-Woong Kim, Seung-Jae Lee, Cheol-Young Park, et al. An experimental study of a circular cylinder's two-degree-of-freedom motion induced by vortex. International Journal of Naval Architecture and Ocean Engineering 2016, 8: 330-343.

24. Narakorn Srinil, Hossein Zanganeh, Alexander Day. Two-degree-of-freedom VIV of circular cylinder with variable natural frequency ratio: Experimental and numerical investigations. Ocean Engineering 2013, 73 (2013) 179-194. 